\title{
Recent developments in the molecular characterization and treatment of oligodendroglial tumors
}

\author{
Martin van den Bent, ${ }^{1}$ Olivier-Louis Chinot, and J. Gregory Cairncross \\ Department of Neuro-Oncology, Dr. Daniel den Hoed Cancer Center, 3008 AE Rotterdam, \\ The Netherlands (M.v.d.B.); Hôpital de la Timone, Service de Neurochirurgie, Boulevard Jean Moulin, 13385 \\ Marseille, France (O.-L.C.); Department of Clinical Neurosciences, University of Calgary and Foothills \\ Medical Centre, 1403 29th Street N.W., Calgary, Alberta T2N 2T9, Canada (S. G.C.)
}

\begin{abstract}
Although many patients with oligodendrogliomas (ODs) and oligoastrocytomas (OAs) benefit from a combination of surgery and adjuvant radiotherapy, most patients eventually experience recurrence of their disease. Recent evidence has shown that ODs are more chemosensitive than other gliomas, including astrocytomas or glioblastoma multiforme. These initial findings have prompted further study of chemotherapy in treating ODs and mixed OAs. Advances in molecular genetic analysis have led to improvements in predicting response to chemotherapy and prognosis for ODs, OAs, and astrocytomas. Pure ODs are more chemosensitive than mixed ODs. This difference is related to different proportions of $1 \mathrm{p} / 19 \mathrm{q}$ loss of heterozygosity in these neoplasms. Therefore, genetic analysis is likely to be key in determining appropriate treatment. The most common first-line chemotherapy for patients with OD is a procarbazine, lomustine, and vincristine (PCV) combination regimen. However, this regimen is associated with cumulative myelosuppression, nausea,
\end{abstract}

Received July 9, 2002; accepted August 6, 2002.

${ }^{1}$ Address correspondence and reprint requests to Martin van den Bent, Department of Neuro-Oncology, Dr. Daniel den Hoed Cancer Center, P.O. Box 5201, 3008 AE Rotterdam, The Netherlands

${ }^{2}$ Abbreviations used are as follows: $\mathrm{AA}$, anaplastic astrocytoma; $\mathrm{AOA}$, anaplastic oligoastrocytoma; AOD, anaplastic oligodendroglioma; CBTRUS, Central Brain Tumor Registry of the United States; EGFR, epidermal growth factor receptor; EORTC, European Organization for Research and Treatment of Cancer; GBM, glioblastoma multiforme; GFAP, glial fibrillary acidic protein; MTIC, 5-(3-N-methyltriazen-1-yl)imidazole-4-carboxamide; OA, oligoastrocytoma; OD,

oligodendroglioma; PCV, procarbazine, lomustine, and vincristine; PCV-I, intensified procarbazine, lomustine, and vincristine; PR, partial response; RT, radiotherapy; SD, stable disease. vomiting, and weight loss. Therefore, other chemotherapy agents and regimens have been investigated. Perhaps the most promising is temozolomide, a novel alkylating agent that freely crosses the blood-brain barrier. Temozolomide is approved in the United States for the treatment of recurrent anaplastic astrocytomas and in Europe for any recurrent high-grade gliomas. Initial reports suggest that temozolomide is effective in treating ODs as first- and second-line chemotherapy. Unlike the PCV regimen, temozolomide is not associated with cumulative myelosuppression and is usually well tolerated. Further studies are needed to confirm the efficacy and safety profile of temozolomide and to determine the optimal dose and schedule for treating ODs. Neuro-Oncology 5, 128-138, 2003 (Posted to Neuro-Oncology [serial online], Doc. 02-028, February 18, 2003. URL http://neuro-oncology.mc.duke. edu)

$\mathrm{P}$ rimary brain tumors compose approximately $1 \%$ of new cancer cases in the United States, with an estimated 17,200 new cases in 2001 (Greenlee et al., 2001). Given the U.S. mortality rate of 4.1 per 100,000 persons, it is estimated that brain tumors accounted for approximately 13,100 deaths in 2001 (Greenlee et al., 2001; Ries et al., 2001). In adults, the most common brain tumors are anaplastic astrocytoma $(\mathrm{AA})^{3}$ and glioblastoma multiforme (GBM) (Burger et al., 1985; Kleihues et al., 1993). Of all the brain tumors reported to the Central Brain Tumor Registry of the United States (CBTRUS) between 1992 and 1997, 3.5\% were oligodendrogliomas (ODs) (CBTRUS, 2001). This is in agreement with initial estimates that suggested that approximately $4 \%$ of primary brain tumors were ODs (Mork et al., 1985). However, recent changes in histologic cri- 
teria (distinguishing ODs and oligoastrocytomas [OAs] from diffuse and pilocytic astrocytomas) have increased this estimate to $25 \%$ (Coons et al., 1997).

In general, malignant gliomas are resistant to chemotherapy. However, reports in the late 1980s suggested that ODs are more chemosensitive than astrocytomas or GBMs (Cairncross and Macdonald, 1988; Cairncross and Macdonald, 1991). Additional studies have confirmed these initial findings, with reports showing that roughly $60 \%$ to $70 \%$ of ODs are sensitive to a combination regimen of procarbazine, lomustine, and vincristine (PCV) (Allison et al., 1997; Cairncross et al., 1994; Kyritsis et al., 1993; van den Bent et al., 1998). However, the PCV regimen is associated with cumulative myelosuppression, nausea, vomiting, and weight loss. In addition, all patients who respond to PCV ultimately experience relapse. Recently, other chemotherapy agents, including carboplatin, cisplatin, etoposide, paclitaxel, and temozolomide, have been investigated as second-line chemotherapy for the treatment of OD. Temozolomide is a new oral alkylating agent that has been approved in the United States for the treatment of refractory AA and in Europe for recurrent AA and GBM. This novel agent with noncumulative and manageable myelosuppression has also shown promise in the treatment of OD.

Herein we review the importance of distinguishing ODs from astrocytomas and mixed astrocytic tumors. In addition, we discuss the effectiveness of first- and secondline chemotherapy in the treatment of OD, with a focus on temozolomide.

\section{Clinical Features of Oligodendrogliomas}

Several genetic markers (i.e., specific chromosomal abnormalities) have recently been identified that are characteristic of ODs; however, histologic markers that can definitively distinguish ODs from astrocytomas or mixed OAs remain elusive. Histologic criteria for distinguishing ODs from astrocytomas remain subjective, with significant interobserver variability (Coons et al., 1997; Mork et al., 1985; Smith et al., 1999). Nevertheless, advances in genetic analysis have improved the identification of and distinction between these gliomas. The following is a discussion of the known clinical, histologic, and genetic characteristics of low-grade OD, anaplastic oligodendroglioma (AOD), and anaplastic oligoastrocytoma (AOA).

\section{Low-grade Oligodendrogliomas}

Low-grade (WHO grade II) ODs typically emerge in patients $<40$ years of age. These tumors are slow growing and are typically located supratentorially and in the frontal lobe. On MRI, low-grade ODs show increased signal intensity on $\mathrm{T} 2$-weighted images without enhancement. On CT, these tumors appear as low-density masses with no enhancement and may exhibit calcification. The presence of calcification is suggestive of but not specific for OD. Before the widespread use of MRI, approximately $70 \%$ of patients presented with either generalized or focal seizures of long-standing duration with an antecedent history of 7 to 8 years (Celli et al., 1994; Olson et al., 2000). The remainder of patients presented with signs of increased intracranial pressure, focal deficits, or changes in mental state. The prognosis for those patients with increased intracranial pressure or focal deficit is similar to the prognosis for patients with AA (Celli et al., 1994; Daumas-Duport et al., 1997a).

Histologically, low-grade ODs are characterized by uniformly round to oval cells with round nuclei and bland chromatin. The cell density is usually low to moderate. The morphology of these cells is often referred to as the "fried egg appearance" because of a perinuclear halo. A delicate network of branching blood vessels (chicken-wire pattern) is also indicative of low-grade OD. In addition, these tumors often demonstrate perineuronal satellitosis and microcalcifications (Bauman and Cairncross, 2001; Reifenberger et al., 2000). Oligodendrogliomas rarely exhibit the sparse glial fibrillary acidic protein (GFAP) staining that is usually due to the presence of reactive astrocytes. However, ODs occasionally express GFAP; therefore, GFAP staining alone is not a reliable assay to rule out OD. Coons et al. (1997) suggested that the classic histologic features described above are not sufficient for the differential diagnosis of OD, and therefore OD may often be misdiagnosed as AA or GBM. These authors suggested that when histologic features are used to diagnose OD, special emphasis should be given to identifying other signs, such as microgemistocytes, gliofibrillary oligodendrocytes, and protoplasmic astrocytes, which may suggest that the tumor is of oligodendroglial origin.

Advances in genetic analysis have provided an opportunity to improve the differential diagnosis of OD. The most frequently reported chromosomal lesions in OD are allelic loss of the 1p and 19q loci (Kraus et al., 1995; Reifenberger et al., 1994), as shown in Table 1. The incidence of either $1 \mathrm{p}$ or $19 \mathrm{q}$ chromosomal deletions in OD is reported to be approximately $75 \%$ (Cairncross et al., 1998; Smith et al., 1999), and the combined loss of $1 \mathrm{p}$ and $19 \mathrm{q}$ has been reported to occur in $60 \%$ to $70 \%$ of all ODs (van den Bent, 2000). In contrast, this combination of chromosomal lesions is present in $<11 \%$ of tumors thought to be of astrocytic origin because of their microscopic appearance (Maintz et al., 1997; Smith et al., 1999, 2000). Bigner et al. (1999) reported that the loss of heterozygosity for $1 p$ and $19 q$ was seen in 17 of $23(74 \%)$ well-differentiated ODs, whereas only 3 of $8(38 \%)$ OAs exhibited loss of heterozygosity for $1 \mathrm{p}$ and $19 \mathrm{q}$. Moreover, these chromosomal abnormalities have prognostic significance: ODs with combined $1 p$ and $19 q$ deletions are more likely to respond to chemotherapy (Cairncross et al., 1998; van den Bent, 2003b), and the presence of $1 \mathrm{p}$ deletions is associated with longer progression-free survival after radiotherapy (RT) or chemotherapy compared with the survival associated with other chromosomal abnormalities, including TP53 mutations observed in many tumors of astrocytic origin (Bauman et al., 2000). Studies that have combined morphology and genetic analysis suggest that oligodendroglial tumors with a classical histologic appearance (perinuclear halo, chicken-wire vascular pattern) are associated with the loss of $1 \mathrm{p} / 19 \mathrm{q}$, whereas in tumors with an atypical appearance, other chromosomal abnormalities are found 
Table 1. Common molecular genetic abnormalities, tumor grade, and estimated 5 -year survival rate in glioma subtypes

\begin{tabular}{|c|c|c|c|}
\hline Glioma subtype & Genetic abnormalities $^{\mathrm{a}}$ & $\begin{array}{l}\text { WHO tumor } \\
\text { grade }\end{array}$ & $\begin{array}{l}5 \text {-year survival } \\
\text { rate, }{ }^{\mathrm{b}}\end{array}$ \\
\hline Oligodendroglioma & $\begin{array}{l}\text { Chromosome } 1 \mathrm{p} \text { loss } \\
\text { Chromosome } 19 \mathrm{q} \text { loss }\end{array}$ & II & 62 \\
\hline Anaplastic oligodendroglioma & $\begin{array}{l}\text { Chromosome } 1 p \text { loss and/or chromosome } 19 q \text { loss plus: } \\
\text { Chromosome } 9 p \text { loss } \\
\text { CDKN2A mutations } \\
\text { Chromosome } 10 \text { loss } \\
\text { Chromosome } 7 \text { amplification }\end{array}$ & III & 38 \\
\hline Oligoastrocytoma & TP53/chromosome $17 p$ loss & II and III & 56 \\
\hline Astrocytoma & $\begin{array}{l}\text { TP53 mutation/chromosome } 17 \mathrm{p} \text { loss } \\
\text { Chromosome } 22 \mathrm{q} \text { loss }\end{array}$ & II & 45 \\
\hline Anaplastic astrocytoma & $\begin{array}{l}\text { TP53 mutation/chromosome } 17 \mathrm{p} \text { loss or chromosome } \\
22 \mathrm{q} \text { loss plus: } \\
\text { CDKN2A and CDKN2B deletion/chromosome } 9 \mathrm{p} \text { loss } \\
\text { Chromosome } 19 \mathrm{q} \text { loss } \\
\text { Chromosome } 11 \mathrm{p} \text { loss }\end{array}$ & III & 28 \\
\hline Glioblastoma multiforme & $\begin{array}{l}\text { PTEN mutation/chromosome } 10 \mathrm{q} \text { loss } \\
\text { EGFR amplification and rearrangement }\end{array}$ & IV & 3 \\
\hline
\end{tabular}

Abbreviation: EGFR, epidermal growth factor receptor.

aLouis and Cavenee, 2001.

${ }^{\mathrm{b}}$ CBTRUS, 2001.

(e.g., TP53 mutations) (Sasaki et al., 2002; Watanabe et al., 2002; van den Bent et al., 2003b). In a series of lowgrade tumors, the genotype predicted response to chemotherapy better than the presence or absence of classical histologic OD features (Sasaki et al., 2002). However, there is evidence that the reverse can also be true; some atypical ODs have $1 \mathrm{p} / 19 \mathrm{q}$ loss of heterozygosity, and some typical OD tumors do not show these lesions (Sasaki et al., 2002). Nevertheless, a loosening of the histologic criteria for an OD diagnosis, as proposed by some, will likely result in a heterogenous tumor population in terms of genetic analysis (Coons et al., 1997). The anatomic location of ODs also seems to correlate with the genotype. Oligodendrogliomas with $1 \mathrm{p} / 19 \mathrm{q}$ loss of heterozygosity tend to occur in the frontal, parietal, and occipital lobes, and have a tendency for widespread growth across the midline (Zlatescu et al., 2001). They also tend to present with seizures (in contrast to focal deficits) and with a low-grade tumor (van den Bent et al., 2003b).

Patients with low-grade OD (WHO grades I and II) have a median survival of 10 to 17 years (Leighton et al., 1997; Olson et al., 2000; Shaw et al., 1992) and a 5-year survival rate of approximately $75 \%$. In addition, patients with low-grade OD or OA experience longer overall survival compared with patients with low-grade astrocytoma (13 vs. 7.5 years, $P=0.003$ ) (Leighton et al., 1997). Despite this favorable clinical prognosis, virtually all low-grade ODs will eventually recur, usually after malignant transformation to AOD (WHO grade III).

\section{Anaplastic Oligodendrogliomas}

De novo AODs typically occur in patients $>40$ years of age (Daumas-Duport et al., 1997a). These tumors are clinically aggressive and usually present following a short duration of acute symptoms including focal neurologic deficits or symptoms of mass effect (Daumas-Duport et al., 1997b; Dehghani et al., 1998). Anaplastic oligodendrogliomas are characterized by high cellularity, high mitotic activity, microvascular proliferation, nuclear abnormalities, and necrosis (WHO grade III) (Bauman and Cairncross, 2001; Reifenberger et al., 2000). On MRI or CT, these tumors are characterized by enhancement, which is thought to be the macroscopic equivalent of microvascular proliferation (Bauman and Cairncross, 2001). A number of histologic features, including cell density (Mork et al., 1985), the number of mitoses (Burger et al., 1987), the presence of endothelial hyperplasia (Daumas-Duport et al., 1997b), necrosis (Burger et al., 1987; Gannett et al., 1994; Mork et al., 1985), and the presence of pleomorphism (Smith et al., 1999), have all been identified as important prognostic factors for AOD. Several studies showed endothelial hyperplasia to be the strongest and most independent variable in prognosis compared with the other histologic characteristics (Daumas-Duport et al., 1997a; Giannini et al., 2001).

Genetic analysis has shown that in addition to the allelic loss of $1 \mathrm{p}$ and $19 \mathrm{q}$, AODs often exhibit additional chromosomal deletions (Table 1 ). Loss of heterozygosity for $9 \mathrm{p}$ and/or deletion of the CDKN2A gene occurs in $33 \%$ to $42 \%$ of AODs, and deletions on chromosome 10 occur in $19 \%$ to $25 \%$ of cases (Bigner et al., 1999; Reifenberger et al., 1994). Despite a clear oligodendroglial morphology, some AOD tumors display a combination of chromosome 7 amplification and chromosome 10 deletions, which is more characteristic of an astrocytic lineage (Bigner et al., 1999; Jeuken et al., 1999). Amplification of the epidermal growth factor receptor (EGFR) has also recently been described in $20 \%$ to $25 \%$ of AODs (Chinot et al., 2001a; Hoang-Xuan et al., 2001). Both the loss of $10 \mathrm{q}$ and the amplification of EGFR are inversely related to $1 \mathrm{p} / 19 \mathrm{q}$ loss, and both are associated with poor chemosensitivity and short survival (Chinot et al., 2001a; 
Hoang-Xuan et al., 2001; Sasaki et al., 2001). Studies investigating PTEN mutations have provided conflicting results. Sasaki et al. (2001) reported 7 PTEN mutations in 72 cases, with a combined loss of $10 \mathrm{q}$ in 6 of these tumors. In contrast, Jeuken et al. (2000) and HoangXuan et al. (2001) reported no PTEN mutations in oligodendroglial tumors. The mutually exclusive occurrence of $1 \mathrm{p} / 19 \mathrm{q}$ loss and TP53 mutations, EGFR amplification, 10q loss, or PTEN mutations suggests that these tumors are in fact derived from different precursor cells. This hypothesis is supported by the marked differences in the response to treatment and the prognosis for these genetically diverse tumors.

It has been suggested that the single most important prognostic factor affecting progression-free and overall survival in patients with OD is the pathologic tumor grade (Allam et al., 2000; Shaw et al., 1992). Patients with AOD have a substantially worse prognosis than do patients with low-grade OD, with reported median survivals of 4 to 5 years and a 5 -year survival rate of only approximately $40 \%$ (Shaw et al., 1992, 1997). However, $\mathrm{AOD}$ is associated with a better prognosis than other anaplastic gliomas; patients with AA survive for 2 to 3 years, and patients with GBM survive for $<2$ years (Louis and Cavenee, 2001). Winger et al. (1989) reported that in 285 patients with supratentorial anaplastic gliomas, patients with AOD had the longest median survival time (5.3 years), compared with patients with GBM, AA, and AOA.

\section{Oligoastrocytomas}

The existence of the OA subtype is a matter of controversy. Mixed tumors have morphologic characteristics of both astrocytic tumors and pure ODs. These tumors are generally associated with tumoral oligodendroglial foci and gemistocytic astrocytic components mixed within the same region of the tumor. However, unlike pure ODs, OAs typically have areas that are GFAP positive (Reifenberger et al., 2000). This staining pattern may be due to the astrocytic component of the tumor but may also be due to reactive astrocytes. Some centers consider a tumor to be an OA if $\geq 10 \%$ of the cells are astrocytic in origin (Krouwer et al., 1997). In contrast, the European Organization for Research and Treatment of Cancer (EORTC) and the Radiation Therapy Oncology Group have defined $\mathrm{OA}$ as an astrocytic tumor that contains $\geq 25 \%$ oligodendroglial elements (van den Bent, 2000). However, these are arbitrary definitions that may have no biologic or clinical relevance. Attempts to establish clear-cut definitions are confounded by the fact that the percentage of astrocytic and oligodendroglial elements is subject to significant interobserver variation (Krouwer et al., 1997). Therefore, there is considerable disagreement with regard to the diagnosis of an OA based on the percentage of either astrocytic or oligodendrocytic components (Bigner et al., 1999; Krouwer et al., 1997).

Clinically, there does appear to be a distinction between OA and pure astrocytoma. The prognosis for patients with $\mathrm{OA}$ is better than the prognosis for patients with astrocytoma (Donahue et al., 1997; Krouwer et al.,
1997; Leighton et al., 1997; Shaw et al., 1994). In contrast, patients with OA have a worse prognosis than patients with OD. Patients with OA have 5- and 10-year survival rates of $63 \%$ and $33 \%$, respectively, whereas patients with so-called pure OD have 5- and 10-year survival rates of $75 \%$ and $46 \%$, respectively, although this difference is moderate and not constant (Shaw et al., 1992). Nevertheless, the explanation for these differences may lie in the molecular heterogeneity of mixed tumors.

Maintz et al. (1997) have recently provided genetic evidence that OAs are not mixed tumors at all, but rather tumors of either oligodendroglial or astrocytic origin. In this study, OAs fell into 2 categories: those that had $1 \mathrm{p}$ and $19 \mathrm{q}$ deletions, typical of ODs, and those that had TP53 mutations, typical of astrocytomas (Table 1). Bigner et al. (1999) reported that among 55 OD, AOD, and AOA tumors, 14 had $17 \mathrm{p}$ loss of heterozygosity and/or TP53 mutations, and the majority of these 14 tumors had significant astrocytic histologic elements. Only 1 of the 14 tumors with $17 \mathrm{p}$ loss of heterozygosity and/or TP53 mutations also had $1 \mathrm{p}$ and $19 \mathrm{q}$ loss of heterozygosity. These data further support the idea that OAs are of either oligodendroglial or astrocytic lineage.

Clinical observations appear to correlate with the genetic profile of these tumors. Patients with OD and $1 \mathrm{p} / 19 \mathrm{q}$ deletions have higher survival rates, which can probably be attributed to the sensitivity of these tumors to treatment, particularly chemotherapy, and the less aggressive clinical behavior of these tumors. Similarly, the lower survival rates in patients with OA can partially be explained by the presence of more unfavorable prognostic factors in tumors without $1 \mathrm{p} / 19 \mathrm{q}$ lesions. Similar observations have been made in patients with AOD. Among 50 patients diagnosed with AOD, 23 patients who had $1 \mathrm{p} / 19 \mathrm{q}$ deletions had a higher response rate $(100 \%)$ to chemotherapy and a longer median survival (123 months) than 8 patients with TP53 mutations (33\% response rate; 71-month median survival) (Ino et al., 2001).

New developments may provide more specific molecular markers for tumors of true oligodendroglial lineage. There is accumulating evidence that suggests that oligodendroglial tumors arise from O2-A oligodendroglial progenitor cells. Both the $\mathrm{O} 2-\mathrm{A}$ progenitor cell and oligodendroglial tumors coexpress NG2 chondroitin proteoglycan and platelet-derived growth factor receptor- $\alpha$. The combined expression of these 2 proteins is not observed in normal brain tissue or GBM (Shoshan et al., 1999). The recently discovered OLIG1/2 basic helix-loop-helix transcription factors are the earliest known markers of oligodendrocyte precursor cells. These transcription factors are also expressed in ODs but not in astrocytic tumors (Lu et al., 2001; Marie et al., 2001). These and other as yet unidentified markers may eventually be used to determine treatment options and predict prognosis.

In summary, histologic characterization appears insufficient to clearly distinguish glioma subtypes. Recent advances in molecular genetics have improved the differential diagnosis of these tumors. These advances have initiated discussions on reevaluating the current subtype classification of gliomas based on histology and the cel- 
lular origin of the major component of the tumor in favor of a system based on genetic markers (Bigner et al., 1999; Ino et al., 2001). This reevaluation has led to an increasing proportion of gliomas being identified as oligodendroglial tumors. With further advances in genetic analysis, the OA tumor subtype may disappear and be replaced with a diagnosis of either OD or astrocytoma. Further, molecular genetic characterization of glioma subtypes may provide better guidance in selecting the appropriate treatment option for these patients. Eventually, genetic analysis may result in individualized rational treatments that are tailored to tumors with specific genetic abnormalities.

\section{Treatment of Oligodendrogliomas}

\section{Surgery}

Surgical resection is the primary treatment modality for patients with operable tumors and provides tissue for the classification, grading, and genetic analysis of the tumor. In addition, debulking surgery can relieve neurologic deficits and symptoms due to mass effect and can improve seizure control. In low-grade glioma, one uncontrolled study demonstrated that complete resection is associated with long disease-free intervals (Berger et al., 1994). In another study, OD patients who underwent subtotal resection had improved survival, although many of these patients were young and had low-grade tumors (Celli et al., 1994; Schiffer et al., 1997; Shaw et al., 1994). In contrast, other studies have shown that gross total resection in patients with OD provided no survival benefit (DaumasDuport et al., 1997b; Kros et al., 1994). Nonetheless, given the potential clinical benefits associated with surgical resection, the tumor should be resected as extensively as is safely possible whenever warranted.

\section{Radiotherapy}

Surgical resection followed by adjuvant RT is currently the standard of care for patients with high-grade gliomas. Randomized trials comparing adjuvant RT and best standard of care after surgery have confirmed that adjuvant RT provides significant yet modest improvements in survival among these patients (Walker et al., 1978, 1980). However, the value of RT in patients with low-grade OD or OA has not been studied in controlled, randomized trials and remains controversial. Some retrospective studies have supported postoperative RT in patients with OD (Gannett et al., 1994; Wallner et al., 1988). Some studies suggest that RT provides a survival benefit in patients with OD, mainly in the subgroup of patients with neurologic deficit or for whom surgery was limited to biopsy or partial surgery (Celli et al., 1994; Shaw et al., 1992), whereas other studies have reported no benefit of postoperative RT in the treatment of OD (Bullard et al., 1987; Nijjar et al., 1993; Reedy et al., 1983). Until controlled, randomized studies are conducted, a prudent approach for treating patients with low-grade OD who can undergo complete resection may be to observe the patient and withhold RT until progression. An important rationale for the deferral of RT is provided by compelling data demonstrating pronounced cognitive deficits in patients with low-grade gliomas who have undergone early RT (Surma-aho et al., 2001; Taphoorn et al., 1994). In contrast, patients with large, unresectable, or incompletely resected tumors; focal deficits; anaplastic tumors; or enhancing lesions should be treated without delay (Macdonald, 1994). Radiotherapy is the standard treatment approach, although first-line chemotherapy is also an option. Further, advances in the genetic characterization of ODs may also facilitate the decision-making process for the timing of RT.

\section{Chemotherapy in Low-grade Oligodendrogliomas}

Data on the use of chemotherapy in the treatment of lowgrade OD and OA are limited, and the role of chemotherapy in this patient population remains unclear. Although chemotherapy is generally proposed for symptomatic aggressive tumors (i.e., with focal deficits) that behave like anaplastic tumors, there are mixed reports on the efficacy of chemotherapy in low-grade OD. Further, because of the nonenhancing characteristic of low-grade tumors, it is difficult to assess response. More studies in OD patients with nonenhancing indolent tumors, symptoms limited to seizures, and a more favorable prognosis are needed. Nevertheless, several small studies have investigated the PCV regimen for the treatment of patients with low-grade OD. Paleologos et al. (1994) reported that 3 of $5(60 \%)$ patients with nonenhancing grade I/II OD responded to PCV. These patients had not received prior RT and were not receiving steroids. Mason et al. (1996) reported that all 9 patients with low-grade OD treated with PCV improved by clinical or MRI criteria, and no patients experienced deterioration while receiving therapy. Soffietti et al. (1999) investigated the use of PCV in a group of chemotherapy-naive patients with low-grade, nonenhancing OD or OA. Of 13 newly diagnosed patients, $3(23 \%)$ achieved a partial response (PR) and 10 $(77 \%)$ achieved stable disease (SD). In this same study, 2 of $7(29 \%)$ patients with recurrent low-grade OD achieved a PR and 5 (71\%) achieved SD. Recently, van den Bent et al. $(2002 \mathrm{a})$ reported that of 12 patients treated with the standard PCV regimen, 9 showed either clinical or radiological improvement. Therefore, in patients with lowgrade OD, the PCV regimen appears to be effective. However, larger, prospective studies and standardization of efficacy end points are required to fully evaluate the effectiveness of the PCV regimen in this patient population.

\section{Chemotherapy in Anaplastic Oligodendrogliomas and Oligoastrocytomas}

The first demonstration that AODs are sensitive to chemotherapy was reported by Cairncross and Macdonald (1988). The PCV regimen was shown to be effective in AOD, which prompted further studies. The PCV regimen is the most common chemotherapy used to treat AOD and AOA, and has been investigated as both firstand second-line chemotherapy (first and second recur- 
rence after surgery and RT). Other agents, including carboplatin, paclitaxel, CPT-11, and temozolomide, have also been investigated as second-line chemotherapy.

First-line chemotherapy with PCV. Several single-institution studies have demonstrated high response rates with PCV at first recurrence. Cairncross and Macdonald (1991) reported that all 7 patients with AOD who were treated with PCV responded to treatment, with median response duration of 14 months (Table 2). Their subsequent phase 2, multicenter trial of intensified PCV (PCV-I) in first-relapsed patients with AOD demonstrated a $75 \%$ response rate among eligible patients (tumors with $>90 \%$ oligodendroglial origin), a median time to progression of $\geq 16$ months for the entire group and $\geq 25$ months for complete responders (Table 2) (Cairncross et al., 1994). However, hematologic toxicity was substantial, and dose reductions and delays due to toxicity were frequent. Five patients had serious or unexpected adverse reactions possibly related to PCV-I. Grade 3/4 neutropenia and thrombocytopenia were observed in $40 \%$ and $15 \%$ of cycles, respectively. Vincristine-associated side effects were common $(70 \%$ of patients experiencing distal numbness or paresthesia). Although the PCV-I regimen was very active in this patient population, it proved to be a debilitating regimen.

Van den Bent et al. (1998) reported a 63\% overall response rate among $43 \mathrm{AOD}$ and $9 \mathrm{AOA}$ previously irradiated patients in first relapse with tumors exhibiting $\geq 25 \%$ of oligodendroglial elements (Table 2 ). The median survival time for these patients was 20 months, and the median duration of response was 25 months and 12 months for complete and partial responders, respectively. In this study, $31 \%$ of patients experienced grade $3 / 4$ hematologic toxicities: $25 \%$ and $13 \%$ of patients experienced grade $3 / 4$ neutropenia and thrombocytopenia, respectively, and $6 \%$ of patients experienced grade 3 anemia. Similarly, Streffer et al. (2000) reported an overall response rate of $55 \%$ with a median progression-free survival of $\geq 18$ months in a mixed patient population that included first-relapse (after irradiation) patients with AOA, AOD, and some low-grade OD and OA (Table 2). All of these studies suggest a role for PCV in treating AOD and AOA. However, the cumulative hematologic toxicity and gastrointestinal side effects associated with PCV often limit the duration of its administration.

Second-line chemotherapy. Although the majority of patients respond to first-line PCV, most patients with AOD experience a relapse within 2 years. The possibilities for repeated use of PCV are limited because of cumulative myelosuppression and acquired tumor resistance (Paleologos et al., 1999; Shaw et al., 1992, 1994; Soffietti et al., 1998). Peterson et al. (1996) reported an overall response of only $19 \%$ when chemotherapy was administered after previous neoadjuvant (i.e., prior to RT), first- or secondline PCV therapy. Of the 6 patients who received PCV as neoadjuvant therapy, $33 \%$ achieved an overall response to second-line chemotherapy. Of the 6 patients who received second-line PCV therapy after non-nitrosourea-based primary therapy, 5 patients $(83 \%)$ achieved an overall response. Therefore, not unexpectedly, second-line PCV chemotherapy was more effective in patients who had not received a nitrosourea-based first-line chemotherapy regimen or PCV as neoadjuvant therapy.

Only a few agents, including paclitaxel, CPT-11, carboplatin, etoposide plus cisplatin, and temozolomide, have been investigated for second-line chemotherapy in patients with $\mathrm{AOD}$, and response rates are generally low (Table 3). Of 20 patients previously treated with surgery, RT, and PCV, $3(15 \%)$ obtained a PR and $7(35 \%)$ achieved SD when treated with paclitaxel (Chamberlain and Kormanik, 1997). Median duration of response and $\mathrm{SD}$ in these patients was 9 months (range, 4 to 10 months). All patients had progressive disease at 12 months. Toxicity was primarily hematologic; $10 \%$ of patients had grade 3 anemia, and $30 \%$ and $15 \%$ of patients experienced grade 3/4 thrombocytopenia and neutropenia, respectively. Similarly, 2 of $15(13 \%)$ treated patients responded to CPT-11, and 33\% of these patients remained free from disease progression at 6 months (Chamberlain, 2001). However, treatment with CPT-11 and paclitaxel is complicated by increased drug metabolism due to the induction of cytochrome P450 by many antiepileptic drugs. Likewise, 3 of 23 patients (13\%) treated with second-line carboplatin achieved a response, and only 1 of these responding patients was free of disease progression at 12 months (Nobile et al., 2001). In addition, 4 of 10 patients treated with cisplatin and etoposide as secondline chemotherapy after first-line PCV experienced an overall response; however, none of the responding patients was progression-free at 12 months (Peterson et al., 1996).

Second-line PCV therapy is effective when administered after non-nitrosourea-based first-line therapy; however, most patients are treated with first-line PCV. Further, most second-line therapies following first-line PCV have only moderate response rates and low proportions of patients remaining free from progression at 6 and 12 months. Clearly, there is a need for improved second-line therapies for this patient population.

\section{Clinical Trials with Temozolomide}

Temozolomide, first synthesized by Stevens et al. (1987), is a novel member of the imidazotetrazine family of chemotherapy agents. Like all alkylating agents, this family of compounds has broad antitumor activity. Temozolomide is rapidly and completely absorbed after oral administration, crosses the blood-brain barrier, and achieves effective concentrations in the central nervous system (Marzolini et al., 1998; Stupp et al., 2001). Temozolomide is absorbed as an inactive prodrug. However, on exposure to physiologic $\mathrm{pH}$, temozolomide is spontaneously hydrolyzed to form MTIC (5-[3-N-methyltriazen1-yl]-imidazole-4-carboxamide), the active form of the drug. The cytotoxicity of MTIC is thought to be due primarily to methylation of the $\mathrm{O}^{6}$ and $\mathrm{N}^{7}$ positions of guanine bases in double-stranded DNA.

The ability of temozolomide to cross the blood-brain barrier suggested that it might be effective in treating gliomas. Indeed, temozolomide has been shown to be effective in treating patients with newly diagnosed or 
Table 2. $P C V$ regimen in first-line chemotherapy administered at recurrence after radiotherapy in patients with $A O D$ and $A O A$

\begin{tabular}{|c|c|c|c|c|c|}
\hline \multirow[b]{2}{*}{ Study } & \multirow[b]{2}{*}{ No. of Patients (histology) } & \multicolumn{4}{|c|}{ n (\%) } \\
\hline & & $\overline{C R}$ & PR & OR & SD \\
\hline Cairncross and Macdonald, 1991 & $7(A O D)$ & $2(29)$ & $5(71)$ & $7(100)$ & 0 \\
\hline Cairncross et al., 1994 & $24(\mathrm{AOD})$ & $9(38)$ & $9(38)$ & $18(75)$ & $4(17)$ \\
\hline van den Bent et al., 1998 & $52(43 \mathrm{AOD}, 9 \mathrm{AOA})$ & $9(17)$ & $24(46)$ & $33(63)$ & $10(19)$ \\
\hline Streffer et al., 2000 & 11 (low-grade $O D$, low-grade $O A, A O D$ or $A O A$ ) & $1(9)$ & $5(45)$ & $6(55)$ & $5(45)$ \\
\hline
\end{tabular}

Table 3. Second-line chemotherapy administered at recurrence after first-line PCV in patients with AOD and AOA

\begin{tabular}{|c|c|c|c|c|c|c|c|}
\hline Study & Agent & $\begin{array}{l}\text { No. of } \\
\text { Patients }\end{array}$ & $\begin{array}{l}\text { OR, } \\
\text { n (\%) }\end{array}$ & $\begin{array}{l}\mathrm{SD} \\
\mathrm{n}(\%)\end{array}$ & $\begin{array}{l}\text { MTP, } \\
\text { months }\end{array}$ & $\begin{array}{l}\text { 6-month } \\
\text { PFS (\%) }\end{array}$ & $\begin{array}{l}\text { 12-month } \\
\text { PFS (\%) }\end{array}$ \\
\hline Chamberlain and Kormanik, 1997 & Paclitaxel & 20 & $3(15)$ & $7(35)$ & 4 & 35 & 0 \\
\hline Chamberlain, 2001 & СРТ-11 & 15 & $2(13)$ & $5(33)$ & - & 33 & - \\
\hline Nobile et al., 2001 & Carboplatin & 23 & $3(13)$ & $10(43)$ & 3 & 35 & 4 \\
\hline Peterson et al., 1996 & Etoposide plus cisplatin & 10 & $4(40)$ & $1(10)$ & - & 20 & 0 \\
\hline
\end{tabular}

Abbreviations: $\mathrm{PCV}$, procarbazine, lomustine, and vincristine; $\mathrm{AOD}$, anaplastic oligodendroglioma; $\mathrm{AOA}$, anaplastic oligoastrocytoma; $O \mathrm{R}$, overall response; $S D$, stable disease; $M T P$, median time to progression; PFS, progression-free survival.

Table 4. Second-line temozolomide administered at recurrence after first-line PCV in patients with AOD and AOA

\begin{tabular}{llllllll} 
Study & $\begin{array}{l}\text { No. of } \\
\text { Patients }\end{array}$ & $\begin{array}{l}\text { Response } \\
\text { to PCV (\%) }\end{array}$ & $\begin{array}{l}\text { OR, } \\
\text { n (\%) }\end{array}$ & $\begin{array}{l}\text { SD, } \\
\text { n (\%) }\end{array}$ & $\begin{array}{l}\text { Median } \\
\text { PFS, } \\
\text { months }\end{array}$ & $\begin{array}{l}\text { 6-month } \\
\text { PFS (\%) }\end{array}$ & $\begin{array}{l}\text { 12-month } \\
\text { PFS (\%) }\end{array}$ \\
\hline Chinot et al., 2001b & 48 & 83 & $21(44)$ & $19(40)$ & 6.7 & 50 & 25 \\
van den Bent et al., 2001 & 27 & 38 & $7(26)$ & $8(30)$ & 4 & 44 & 27 \\
van den Bent et al., 2003a & 28 & 50 & $7(23)$ & $9(30)$ & 3.7 & 29 & 11 \\
Costanza et al., 2001 & 34 & 50 & $9(26)$ & $15(44)$ & 6 & 48 & 30 \\
\hline
\end{tabular}

Abbreviations: PCV, procarbazine, lomustine, and vincristine; AOD, anaplastic oligodendroglioma; AOA, anaplastic oligoastrocytoma; OR, overall response; SD, stable disease; PFS, progression-free survival.

recurrent relapsed GBM and AA (Bower et al., 1997; Brada et al., 2001; Brandes et al., 2001; Friedman et al., 1998; Newlands et al., 1996; O’Reilly et al., 1993; Yung et al., 1999, 2000). In a phase 2 trial, Brada et al. (2001) reported a 6 -month progression-free survival rate of $18 \%$ and a median progression-free survival and median overall survival of 2.1 and 5.4 months, respectively, in GBM patients who received temozolomide at first relapse. In patients with AA or AOA, Yung et al. (1999) reported a 6 -month progression-free survival rate of $46 \%$ and a median progression-free survival and median overall survival of 5.4 and 13.6 months, respectively. Temozolomide is currently approved in the United States for the treatment of adult patients with AA refractory to nitrosoureas and procarbazine-containing regimens and in Europe for the treatment of all recurrent high-grade gliomas.

The pivotal trial of temozolomide in 162 patients with recurrent AA included 14 patients with AOA (Yung et al., 1999). Patients in this trial had received prior RT, and 97 patients received adjuvant chemotherapy and were treated with temozolomide at first relapse. Of the 14 patients with AOA, $43 \%$ obtained an objective response to temozolomide treatment with $2(14 \%)$ complete responses, 4 (29\%) PR, and 1 (7\%) SD. Progression-free survival and median overall survival were 5.8 months and 14.8 months, respectively, and the 6-month survival rate for these patients was $79 \%$. Temozolomide treatment was well tolerated; grade $3 / 4$ thrombocytopenia was experienced by only $6 \%$ of patients, and grade $3 / 4$ neutropenia and leukopenia each occurred in only $2 \%$ of patients. Hematologic toxicity was not cumulative.

The activity of temozolomide as second-line chemotherapy after first-line PCV chemotherapy and previous $\mathrm{RT}$ in the treatment of AOA and AOD has been more rigorously studied by Chinot et al. (2001b). They reported an overall response of $44 \%$ in 48 patients with AOD or AOA at second relapse (Table 4). Not unexpectedly, AOD patients had a higher $(49 \%)$ response rate compared with AOA patients (22\%). In addition, AOD patients tended to have longer progression-free survival (7.3 vs. 5.6 months) and longer median overall survival (9.9 vs. 8.7 months) compared with AOA patients. The 6-month and 1-year survival rates for all 48 patients were $77 \%$ and $46 \%$, respectively. For patients with an 
objective response, the median progression-free survival was 13.1 months, and the median overall survival was 16 months. Only $6 \%$ of patients experienced grade $3 / 4$ thrombocytopenia, and no patients experienced grade $3 / 4$ neutropenia. Dose reductions were required in only $1 \%$ of patients, and there were no discontinuations due to treatment-related toxicity.

These encouraging results have recently been confirmed in 2 studies. In a report by van den Bent et al. (2001) on AOD and AOA patients treated with temozolomide, 27 of 30 patients were previously treated with RT and a first-line PCV chemotherapy regimen (Table 4). Of the patients previously treated with PCV, $26 \%$ responded to temozolomide treatment. Further, 2 of 3 chemotherapy-naive patients achieved a complete response. The median time to progression in responding patients was 13 months. Temozolomide was well tolerated in these patients, with grade $3 / 4$ nausea and vomiting and grade $3 / 4$ thrombocytopenia occurring in only $6 \%$ and $2 \%$ of cycles, respectively.

Recently, van den Bent et al. (2003a) reported the results of an EORTC phase 2 study demonstrating a $23 \%$ objective response rate in 30 evaluable AOD or AOA patients treated with temozolomide at recurrence after first-line PCV treatment (Table 4). The median duration of response for these patients was 6.2 months (range, 3 to 15 months). In general, treatment was well tolerated. Four patients developed grade 3/4 nausea and vomiting that was managed with prophylactic antiemetics. Three patients developed grade 3/4 thrombocytopenia, and 1 patient experienced grade 3 leukopenia. Grade 3 dysphagia or stomatitis was observed in 2 patients, and only 1 patient discontinued treatment because of thrombocytopenia. Costanza et al. (2001) reported a response rate of $22 \%$ in a similar patient population (Table 4 ), with $50 \%$ of patients free from disease progression at 6 months.

The lower response rates reported in these studies compared with those reported by Chinot et al. (2001b) might be explained in part by the fact that only $38 \%$ to $50 \%$ of patients enrolled in these trials responded to firstline PCV therapy, whereas $83 \%$ of patients enrolled in the study reported by Chinot et al. responded to first-line PCV treatment. Presumably, differences in response may be explained by different proportions of patients with $1 \mathrm{p} / 19 \mathrm{q}$ loss of heterozygosity status. The correlation between $1 \mathrm{p}$ loss of heterozygosity and sensitivity to PCV suggests that similar studies should be performed to determine if this correlation also occurs in temozolomide-treated patients. Interestingly, these 4 studies investigating the use of second-line temozolomide therapy after prior PCV show $11 \%$ to $46 \%$ of patients free from disease progression at 12 months. These results are superior to those reported with other agents used in secondline therapy in the treatment of AOD or AOA.

\section{Future Role of Temozolomide in the Treatment of Oligodendrogliomas}

Recent studies have shown that treatment with PCV is effective in patients with OD; however, cumulative hema- tologic toxicity remains an issue and limits subsequent treatment options. Further, the use of chemotherapy agents with a poor toxicity profile is not optimal for a relatively indolent disease. Thus, the use of an oral agent such as temozolomide, which is associated with a low incidence of noncumulative hematologic toxicity, may provide added survival benefit without significantly affecting the patient's quality of life.

Several studies have demonstrated the activity of temozolomide in patients with AOD and AOA in both first- and second-line settings. Early results suggest that the efficacy of temozolomide as second-line chemotherapy may be comparable to that of PCV, and that temozolomide is effective as salvage therapy after patients fail first-line PCV. Importantly, although PCV is associated with cumulative myelosuppression, treatment with temozolomide is well tolerated and results in few treatment delays or discontinuations due to myelosuppression. The activity of temozolomide and the proportion of patients remaining free from disease progression at 12 months also compare favorably to results with other single-agent or combination regimens as second-line chemotherapy. Further, the fact that temozolomide is an oral agent with an excellent toxicity profile may increase patient compliance compared with that associated with other agents currently being investigated as second-line therapy (e.g., etoposide/cisplatin, paclitaxel, and carboplatin) that require intravenous administration. As most glioma patients use antiepileptic drugs, the absence of drug interactions between temozolomide and cytochrome P450-inducing agents is another important advantage of temozolomide in this patient population.

At present, there are limited data on the use of temozolomide as first-line chemotherapy in patients with AOD and AOA. However, preliminary results from an EORTC phase 2 study suggest that temozolomide may be as active as PCV in treating patients with oligodendroglial tumors after first relapse (van den Bent et al., 2002 b). Chemotherapy-naive patients with recurrent OD after RT were treated with $200 \mathrm{mg} / \mathrm{m}^{2}$ of temozolomide on days 1 through 5 of a 28 -day cycle. Of 35 evaluable patients, $19(54 \%)$ achieved an objective response, including 9 complete responses and 10 PRs. An additional 10 patients $(29 \%)$ experienced SD. At 12 months, $40 \%$ of patients were free of disease progression. Further studies, including a phase 3 trial comparing temozolomide with PCV, are required to fully elucidate the role of temozolomide in the first-line setting. In addition, temozolomide should be investigated in the adjuvant setting.

The different mechanism of action and improved safety profile of temozolomide in comparison with other agents, including PCV and cisplatin, make it an attractive agent for combination therapy. Other studies are needed to investigate alternative schedules or the combination of other drugs (e.g., paclitaxel or the PCV regimen) with temozolomide. These additional studies may provide evidence of synergistic or additive activity against primary and recurrent OD and OA. 


\section{References}

Allam, A., Radwi, A., El Weshi, A., and Hassounah, M. (2000) Oligodendroglioma: An analysis of prognostic factors and treatment results. Am. J. Clin. Oncol. 23, 170-175.

Allison, R.R., Schulsinger, A., Vongtama, V., Barry, T., and Shin, K.H. (1997) Radiation and chemotherapy improve outcome in oligodendroglioma. Int. J. Radiat. Oncol. Biol. Phys. 37, 399-403.

Bauman, G.S., and Cairncross, J.G. (2001) Multidisciplinary management of adult anaplastic oligodendrogliomas and anaplastic mixed oligoastrocytomas. Semin. Radiat. Oncol. 11, 170-180.

Bauman, G.S., Ino, Y., Ueki, K., Zlatescu, M.C., Fisher, B.J., Macdonald, D.R., Stitt, L., Louis, D.N., and Cairncross, J.G. (2000) Allelic loss of chromosome $1 \mathrm{p}$ and radiotherapy plus chemotherapy in patients with oligodendrogliomas. Int. J. Radiat. Oncol. Biol. Phys. 48, 825-830.

Berger, M.S., Deliganis, A.V., Dobbins, J., and Keles, G.E. (1994) The effect of extent of resection on recurrence in patients with low grade cerebral hemisphere gliomas. Cancer 74, 1784-1791.

Bigner, S.H., Matthews, M.R., Rasheed, B.K.A., Wiltshire, R.N., Friedman, H.S., Friedman, A.H., Stenzel, T.T., Dawes, D.M., McLendon, R.E., and Bigner, D.D. (1999) Molecular genetic aspects of oligodendrogliomas including analysis by comparative genomic hybridization. Am. J. Pathol. $155,375-386$.

Bower, M., Newlands, E.S., Bleehen, N.M., Brada, M., Begent, R.J., Calvert, H., Colquhoun, I., Lewis, P., and Brampton, M.H. (1997) Multicentre CRC phase II trial of temozolomide in recurrent or progressive highgrade glioma. Cancer Chemother. Pharmacol. 40, 484-488.

Brada, M., Hoang-Xuan, K., Rampling, R., Dietrich, P.Y., Dirix, L.Y., Macdonald, D., Heimans, J.J., Zonnenberg, B.A., Bravo-Marques, J.M., Henriksson, R., Stupp, R., Yue, N., Bruner, J., Dugan, M., Rao, S., and Zaknoen, S. (2001) Multicenter phase II trial of temozolomide in patients with glioblastoma multiforme at first relapse. Ann. Oncol. 12, 259-266.

Brandes, A.A., Ermani, M., Basso, U., Amista, P., Berti, F., Scienza, R., Rotilio, A., Pinna, G., Gardiman, M., and Monfardini, S. (2001) Temozolomide as a second-line systemic regimen in recurrent high-grade glioma: $\mathrm{A}$ phase II study. Ann. Oncol. 12, 255-257.

Bullard, D.E., Rawlings, C.E., 3rd, Phillips, B., Cox, E.B., Schold, S.C., Jr., Burger, P., and Halperin, E.C. (1987) Oligodendroglioma. An analysis of the value of radiation therapy. Cancer 60, 2179-2188.

Burger, P.C., Vogel, F.S., Green, S.B., and Strike, T.A. (1985) Glioblastoma multiforme and anaplastic astrocytoma. Pathologic criteria and prognostic implications. Cancer 56, 1106-1111.

Burger, P.C., Rawlings, C.E., Cox, E.B., McLendon, R.E., Schold, S.C., Jr., and Bullard, D.E. (1987) Clinicopathologic correlations in the oligodendroglioma. Cancer 59, 1345-1352.

Cairncross, J.G., and Macdonald, D.R. (1988) Successful chemotherapy for recurrent malignant oligodendroglioma. Ann. Neurol. 23, 360-364.

Cairncross, J.G., and Macdonald, D.R. (1991) Chemotherapy for oligodendroglioma. Progress report. Arch. Neurol. 48, 225-227.

Cairncross, G., Macdonald, D., Ludwin, S., Lee, D., Cascino, T., Buckner, J., Fulton, D., Dropcho, E., Stewart, D., and Schold, C., Jr. (1994) Chemotherapy for anaplastic oligodendroglioma. National Cancer Institute of Canada Clinical Trials Group. J. Clin. Oncol. 12, 2013-2021.

Cairncross, J.G., Ueki, K., Zlatescu, M.C., Lisle, D.K., Finkelstein, D.M., Hammond, R.R., Silver, J.S., Stark, P.C., Macdonald, D.R., Ino, Y., Ramsay, D.A., and Louis, D.N. (1998) Specific genetic predictors of chemotherapeutic response and survival in patients with anaplastic oligodendrogliomas. J. Natl. Cancer Inst. 90, 1473-1479.

CBTRUS (Central Brain Tumor Registry of the United States). Year 2001 Annual Statistics Report: Brain Tumors in the US 1992-1997. Available at http://www.cbtrus.org/2001/2001stats_report.htm
Celli, P., Nofrone, I., Palma, L., Cantore, G., and Fortuna, A. (1994) Cerebral oligodendroglioma: Prognostic factors and life history. Neurosurgery 35 , 1018-1034; discussion 1034-1035.

Chamberlain, M.C. (2001) A phase 1-2 trial of CPT-11 (Camptosar) in the treatment of recurrent intracranial oligodendrogliomas [oligos]. Neurooncol. 3, 356. (Abstract)

Chamberlain, M.C., and Kormanik, P.A. (1997) Salvage chemotherapy with paclitaxel for recurrent oligodendrogliomas J. Clin. Oncol. 15, 3427-3432.

Chinot, O.L., Barrie, M., Dufour, H., Romain, S., Ouafik, L., Figarella, D., Martin, P., and Grisoli, F. (2001a) Resistance of anaplastic oligodendroglioma (AO) to procarbazine-CCNU-vincristine (PCV) is correlate to a glioblastoma phenotype. Proc. Am. Soc. Clin. Oncol. 20, 2060. (Abstract)

Chinot, O.L., Honore, S., Dufour, H., Barrie, M., Figarella-Branger, D., Muracciole, X., Braguer, D., Martin, P.M., and Grisoli, F. (2001b) Safety and efficacy of temozolomide in patients with recurrent anaplastic oligodendrogliomas after standard radiotherapy and chemotherapy. J. Clin. Oncol. 19, 2449-2455.

Coons, S.W., Johnson, P.C., Scheithauer, B.W., Yates, A.J., and Pearl, D.K. (1997) Improving diagnostic accuracy and interobserver concordance in the classification and grading of primary gliomas. Cancer 79, 1381-1393.

Costanza, A., Borgognone, M., Nobile, M., Rudà, R., Mutani, R., and Soffietti, R. (2001) Temozolomide in recurrent oligodendroglial tumors: A phase II study. Neuro-oncol. 3 (Suppl. 1), 66. (Abstract)

Daumas-Duport, C., Varlet, P., Tucker, M.L., Beuvon, F., Cervera, P., and Chodkiewicz, J.P. (1997a) Oligodendrogliomas. Part I: Patterns of growth, histological diagnosis, clinical and imaging correlations: A study of 153 cases. J. Neurooncol. 34, 37-59.

Daumas-Duport, C., Tucker, M.L., Kolles, H., Cervera, P., Beuvon, F., Varlet, P., Udo, N., Koziak, M., and Chodkiewicz, J.P. (1997b) Oligodendrogliomas. Part II: A new grading system based on morphological and imaging criteria. J. Neurooncol. 34, 61-78.

Dehghani, F., Schachenmayr, W., Laun, A., and Korf, H.W. (1998) Prognostic implication of histopathological, immunohistochemical and clinical features of oligodendrogliomas: A study of 89 cases. Acta Neuropathol. (Berl.) 95, 493-504.

Donahue, B., Scott, C.B., Nelson, J.S., Rotman, M., Murray, K.J., Nelson, D.F., Banker, F.L., Earle, J.D., Fischbach, J.A., Asbell, S.O., Gaspar, L.E., Markoe, A.M., and Curran, W. (1997) Influence of an oligodendroglial component on the survival of patients with anaplastic astrocytomas: A report of Radiation Therapy Oncology Group 83-02. Int. J. Radiat. Oncol. Biol. Phys. 38, 911-914.

Friedman, H.S., McLendon, R.E., Kerby, T., Dugan, M., Bigner, S.H., Henry, A.J., Ashley, D.M., Krischer, J., Lovell, S., Rasheed, K., Marchev, F., Seman, A.J., Cokgor, I., Rich, J., Stewart, E., Colvin, O.M., Provenzale, J.M., Bigner, D.D., Haglund, M.M., Friedman, A.H., and Modrich, P.L. (1998) DNA mismatch repair and $0^{6}$-alkylguanine-DNA alkyltransferase analysis and response to Temodal in newly diagnosed malignant glioma. J. Clin. Oncol. 16, 3851-3857.

Gannett, D.E., Wisbeck, W.M., Silbergeld, D.L., and Berger, M.S. (1994) The role of postoperative irradiation in the treatment of oligodendroglioma. Int. J. Radiat. Oncol. Biol. Phys. 30, 567-573.

Giannini, C., Scheithauer, B.W., Weaver, A.L., Burger, P.C., Kros, J.M., Mork, S., Graeber, M.B., Bauserman, S., Buckner, J.C., Burton, J., Riepe, R., Tazelaar, H.D., Nascimento, A.G., Crotty, T., Keeney, G.L., Pernicone, P., and Altermatt, H. (2001) Oligodendrogliomas: Reproducibility and prognostic value of histologic diagnosis and grading. J. Neuropathol. Exp. Neurol. 60, 248-262.

Greenlee, R.T., Hill-Harmon, M.B., Murray, T., and Thun, M. (2001) Cancer 
statistics, 2001 [erratum in CA Cancer J. Clin. 51, 144] CA Cancer J. Clin. 51, 15-36.

Hoang-Xuan, K., He, J., Huguet, S., Mokhtari, K., Marie, Y., Kujas, M., Leuraud, P., Capelle, L., Delattre, J.Y., Poirier, J., Broet, P., and Sanson, $M$. (2001) Molecular heterogeneity of oligodendrogliomas suggests alternative pathways in tumor progression. Neurology 57, 1278-1281.

Ino, Y., Betensky, R.A., Zlatescu, M.C., Sasaki, H., Macdonald, D.R., StemmerRachamimov, A.O., Ramsay, D.A., Cairncross, J.G., and Louis, D.N. (2001) Molecular subtypes of anaplastic oligodendroglioma: Implications for patient management at diagnosis. Clin. Cancer Res. 7, 839-845.

Jeuken, J.W., Sprenger, S.H., Wesseling, P., Macville, M.V., von Deimling, A., Teepen, H.L., van Overbeeke, J.J., and Boerman, R.H. (1999) Identification of subgroups of high-grade oligodendroglial tumors by comparative genomic hybridization. J. Neuropathol. Exp. Neurol. 58, 606-612.

Jeuken, J.W., Nelen, M.R., Vermeer, H., van Staveren, W.C., Kremer, H., van Overbeeke, J.J., and Boerman, R.H. (2000) PTEN mutation analysis in two genetic subtypes of high-grade oligodendroglial tumors. PTEN is only occasionally mutated in one of the two genetic subtypes. Cancer Genet. Cytogenet. 119, 42-47.

Kleihues, P., Burger, P.C., and Scheithauer, B.W. (1993) The new WHO classification of brain tumours. Brain Pathol. 3, 255-268.

Kraus, J.A., Koopmann, J., Kaskel, P., Maintz, D., Brandner, S., Schramm, J., Louis, D.N., Wiestler, O.D., and von Deimling, A. (1995) Shared allelic losses on chromosomes $1 p$ and $19 q$ suggest a common origin of oligodendroglioma and oligoastrocytoma. J. Neuropathol. Exp. Neurol. 54, 91-95.

Kros, J.M., Pieterman, H., van Eden, C.G., and Avezaat, C.J. (1994) Oligodendroglioma: The Rotterdam-Dijkzigt experience. Neurosurgery 34, 959-966; discussion 966.

Krouwer, H.G., van Duinen, S.G., Kamphorst, W., van der Valk, P., and Algra, A. (1997) Oligoastrocytomas: A clinicopathological study of 52 cases. J. Neurooncol. 33, 223-238.

Kyritsis, A.P., Yung, W.K., Bruner, J., Gleason, M.J., and Levin, V.A. (1993) The treatment of anaplastic oligodendrogliomas and mixed gliomas. Neurosurgery 32, 365-370; discussion 371.

Leighton, C., Fisher, B., Bauman, G., Depiero, S., Stitt, L., MacDonald, D., and Cairncross, G. (1997) Supratentorial low-grade glioma in adults: An analysis of prognostic factors and timing of radiation. J. Clin. Oncol. 15, 1294 1301.

Louis, D.N., and Cavenee, W.K. (2001) Neoplasms of the central nervous system. In: DeVita, V.T., Jr., Hellman, S., and Rosenberg, S.A. (Eds.), Cancer: Principles \& Practice of Oncology. 6th ed. Philadelphia: Lippincott Williams \& Wilkins, pp. 2091-2100.

Lu, Q.R., Park, J.K., Noll, E., Chan, J.A., Alberta, J., Yuk, D., Alzamora, M.G., Louis, D.N., Stiles, C.D., Rowitch, D.H., and Black, P.M. (2001) Oligodendrocyte lineage genes (OLIG) as molecular markers for human glial brain tumors. Proc. Natl. Acad. Sci. U.S.A. 98, 10851-10856.

Macdonald, D.R. (1994) Low-grade gliomas, mixed gliomas, and oligodendrogliomas. Semin. Oncol. 21, 236-248.

Maintz, D., Fiedler, K., Koopmann, J., Rollbrocker, B., Nechev, S., Lenartz, D., Stangl, A.P., Louis, D.N., Schramm, J., Wiestler, O.D., and von Deimling, A. (1997) Molecular genetic evidence for subtypes of oligoastrocytomas. J. Neuropathol. Exp. Neurol. 56, 1098-1104.

Marie, Y., Sanson, M., Mokhtari, K., Leuraud, P., Kujas, M., Delattre, J.Y., Poirier, J., Zalc, B., and Hoang-Xuan, K. (2001) OLIG2 as a specific marker of oligodendroglial tumour cells. Lancet 358, 298-300.

Marzolini, C., Decosterd, L.A., Shen, F., Gander, M., Leyvraz, S., Bauer, J., Buclin, T., Biollaz, J., and Lejeune, F. (1998) Pharmacokinetics of temozolomide in association with fotemustine in malignant melanoma and malignant glioma patients: Comparison of oral, intravenous, and hepatic intra-arterial administration [erratum in Cancer Chemother. Pharmacol. 43, 439-440, 1999]. Cancer Chemother. Pharmacol. 42, 433-440.

Mason, W.P., Krol, G.S., and DeAngelis, L.M. (1996) Low-grade oligodendroglioma responds to chemotherapy. Neurology 46, 203-207.

Mork, S.J., Lindegaard, K.F., Halvorsen, T.B., Lehmann, E.H., Solgaard, T., Hatlevoll, R., Harvei, S., and Ganz, J. (1985) Oligodendroglioma: Incidence and biological behavior in a defined population. J. Neurosurg. 63, 881-889.

Newlands, E.S., O'Reilly, S.M., Glaser, M.G., Bower, M., Evans, H., Brock, C., Brampton, M.H., Colquhoun, I., Lewis, P., Rice-Edwards, J.M., Illingworth, R.D., and Richards, P.G. (1996) The Charing Cross Hospital experience with temozolomide in patients with gliomas. Eur. J. Cancer 32A, 2236-2241.

Nijjar, T.S., Simpson, W.J., Gadalla, T., and McCartney, M. (1993) Oligodendroglioma. The Princess Margaret Hospital experience (1958-1984). Cancer 71, 4002-4006.

Nobile, M., Costanza, A., Rudà, R., Mutani, R., and Soffietti, R. (2001) Salvage chemotherapy with carboplatin for recurrent oligodendroglial tumors. Neuro-oncol. 3, 362. (Abstract)

Olson, J.D., Riedel, E., and DeAngelis, L.M. (2000) Long-term outcome of low-grade oligodendroglioma and mixed glioma. Neurology 54, 1442-1448.

O'Reilly, S.M., Newlands, E.S., Glaser, M.G., Brampton, M., Rice-Edwards, J.M., Illingworth, R.D., Richards, P.G., Kennard, C., Colquhoun, I.R., Lewis, P., and Stevens, M.F.G. (1993) Temozolomide: A new oral cytotoxic chemotherapeutic agent with promising activity against primary brain tumours [erratum in Eur. J. Cancer 29A:1500, 1993]. Eur. J. Cancer. 29A, 940-942.

Paleologos, N.A., Vick, N.A., and Kachoris, J.P. (1994) Chemotherapy for lowgrade oligodendrogliomas? Ann. Neurol. 36, 294-295. (Abstract)

Paleologos, N.A., Macdonald, D.R., Vick, N.A., and Cairncross, J.G. (1999) Neoadjuvant procarbazine, CCNU, and vincristine for anaplastic and aggressive oligodendroglioma. Neurology 53, 1141-1143.

Peterson, K., Paleologos, N., Forsyth, P., Macdonald, D.R., and Cairncross, J.G. (1996) Salvage chemotherapy for oligodendroglioma. J. Neurosurg. 85, 597-601.

Reedy, D.P., Bay, J.W., and Hahn, J.F. (1983) Role of radiation therapy in the treatment of cerebral oligodendroglioma: An analysis of 57 cases and a literature review. Neurosurgery 13, 499-503.

Reifenberger, G., Kros, J.M., Burger, P., Louis, D.N., Collins, V.P. (2000) Oligodendroglioma. In: Kleihues, P., Cavanee, W.K. (Eds.), World Health Organization Classification of Tumors: Pathology and Genetics of Tumors of the Nervous System. Lyon: IARC Press, pp. 55-70.

Reifenberger, J., Reifenberger, G., Liu, L., James, C.D., Wechsler, W., and Collins, V.P. (1994) Molecular genetic analysis of oligodendroglial tumors shows preferential allelic deletions on 19q and 1p. Am. J. Pathol. 145, 1175-1190.

Ries, L.A.G., Eisner, M.P., Kosary, C.L., Hankey, B.F., Miller, B.A., Clegg, L., and Edwards, B.K. (Eds.). (2001) SEER Cancer Statistics Review, 1973-1998, Bethesda, Maryland: National Cancer Institute. Available at http://seer. cancer.gov/csr/1973_1998

Sasaki, H., Zlatescu, M.C., Betensky, R.A., Ino, Y., Cairncross, J.G., and Louis, D.N. (2001) PTEN is a target of chromosome 10q loss in anaplastic oligodendrogliomas and PTEN alterations are associated with poor prognosis. Am. J. Pathol. 159, 359-367.

Sasaki, H., Zlatescu, M.C., Betensky, R.A., Johnk, L.B., Cutone, A.N., Cairncross, J.G., and Louis, D.N. (2002) Histopathological-molecular genetic correlations in referral pathologist-diagnosed low-grade "oligodendroglioma." J. Neuropathol. Exp. Neurol. 61, 58-63.

Schiffer, D., Dutto, A., Cavalla, P., Bosone, I., Chio, A., Villani, R., and Bellotti, C. (1997) Prognostic factors in oligodendroglioma. Can. J. Neurol. Sci. 24, 313-319. 
Shaw, E.G., Scheithauer, B.W., O'Fallon, J.R., Tazelaar, H.D., and Davis, D.H (1992) Oligodendrogliomas: The Mayo Clinic experience. J. Neurosurg. 76, 428-434.

Shaw, E.G., Scheithauer, B.W., O'Fallon, J.R., and Davis, D.H. (1994) Mixed oligoastrocytomas: A survival and prognostic factor analysis. Neurosurgery 34, 577-582; discussion 582

Shaw, E.G., Scheithauer, B.W., and O'Fallon, J.R. (1997) Supratentorial gliomas: A comparative study by grade and histologic type. J. Neurooncol. 31, 273-278.

Shoshan, Y., Nishiyama, A., Chang, A., Mork, S., Barnett, G.H., Cowell, J.K., Trapp, B.D., and Staugaitis, S.M. (1999) Expression of oligodendrocyte progenitor cell antigens by gliomas: Implications for the histogenesis of brain tumors. Proc. Natl. Acad. Sci. U.S.A. 96, 10361-10366.

Smith, J.S., Alderete, B., Minn, Y., Borell, T.J., Perry, A., Mohapatra, G., Hosek, S.M., Kimmel, D., O'Fallon, J., Yates, A., Feuerstein, B.G., Burger, P.C., Scheithauer, B.W., and Jenkins, R.B. (1999) Localization of common deletion regions on $1 p$ and $19 q$ in human gliomas and their association with histological subtype. Oncogene 18, 4144-4152.

Smith, J.S., Perry, A., Borell, T.J., Lee, H.K., O'Fallon, J., Hosek, S.M., Kimmel, D., Yates, A., Burger, P.C., Scheithauer, B.W., and Jenkins, R.B. (2000) Alterations of chromosome arms $1 p$ and $19 q$ as predictors of survival in oligodendrogliomas, astrocytomas, and mixed oligoastrocytomas. J. Clin. Oncol. 18, 636-645.

Soffietti, R., Rudà, R., Bradac, G.B., and Schiffer, D. (1998) PCV chemotherapy for recurrent oligodendrogliomas and oligoastrocytomas. Neurosurgery 43, 1066-1073.

Soffietti, R., Rudà, R., Borgognone, M., and Schiffer, D. (1999) Chemotherapy with PCV for low grade nonenhancing oligodendrogliomas and oligoastrocytomas. Neurology 52 (Suppl. 2), A423-A424. (Abstract)

Stevens, M.F., Hickman, J.A., Langdon, S.P., Chubb, D., Vickers, L., Stone, R., Baig, G., Goddard, C., Gibson, N.W., and Slack, J.A. (1987) Antitumor activity and pharmacokinetics in mice of 8-carbamoyl-3-methyl-imidazo[5,1-d]-1,2,3,5-tetrazin-4(3H)-one (CCRG 81045; $M$ \& B 39831), a novel drug with potential as an alternative to dacarbazine. Cancer Res. 47, 5846-5852.

Streffer, J., Schabet, M., Bamberg, M., Grote, E.H., Meyermann, R., Voigt, K., Dichgans, J., and Weller, M. (2000) A role for preirradiation PCV chemotherapy for oligodendroglial brain tumors. J. Neurol. 247, 297302.

Stupp, R., Ostermann, S., Leyvraz, S., Csajka, C., Buclin, T., and Decosterd, L.A. (2001) Cerebrospinal fluid levels of temozolomide as a surrogate marker for brain penetration. Proc. Am. Soc. Clin. Oncol. 20, 59 (Abstract)

Surma-aho, O., Niemela, M., Vilkki, J., Kouri, M., Brander, A., Salonen, O., Paetau, A., Kallio, M., Pyykkonen, J., and Jaaskelainen, J. (2001) Adverse long-term effects of brain radiotherapy in adult low-grade glioma patients. Neurology 56, 1285-1290.

Taphoorn, M.J., Schiphorst, A.K., Snoek, F.J., Lindeboom, J., Wolbers, J.G., Karim, A.B., Huijgens, P.C., and Heimans, J.J. (1994) Cognitive functions and quality of life in patients with low-grade gliomas: The impact of radiotherapy. Ann. Neurol. 36, 48-54.

van den Bent, M.J. (2000) Chemotherapy of oligodendroglial tumours: Current developments. Forum (Genova) 10, 108-118.

van den Bent, M.J., Kros, J.M., Heimans, J.J., Pronk, L.C., van Groeningen, C.J., Krouwer, H.G.J., Taphoorn, M.J.B., Zonnenberg, B.A., Tijssen, C.C., Twijnstra, A., Punt, C.J.A., and Boogerd, W. (1998) Response rate and prognostic factors of recurrent oligodendroglioma treated with procarbazine, CCNU, and vincristine chemotherapy. Dutch Neuro-oncology Group. Neurology 51, 1140-1145.

van den Bent, M.J., Keime-Guibert, F., Brandes, A.A., Taphoorn, M.J.B., Kros,
J.M., Eskens, F.A.L.M., and Carpentier, A.F. (2001) Temozolomide chemotherapy in recurrent oligodendroglioma. Neurology 57, 340-342.

van den Bent, M.J., van der Rijt, C.D.D., Enting, R.H., van de Heuvel, I., Sillevis Smitt, P.A.E., ter Steegh, E., Dijke, I.E., Looijenga, L., and Kros, J.M. (2002a) PCV chemotherapy in newly diagnosed and recurrent low grade oligodendroglial tumors. Neuro-oncol. 4, 378. (Abstract)

van den Bent, M.J., Taphoorn, M.J.B., Brandes, A.A., Menten, J., Stupp, R., Frenay, M., van der Rijt, C.C.D., Kros, J.M., Allgeier, A., and Gorlia, T. (2002b) EORTC brain tumor group study 26971: First line chemotherapy with temozolomide in recurrent oligodendroglial tumors; a phase II study. Proc. Am. Soc. Clin. Oncol. 21, 72. (Abstract)

van den Bent, M.J., Chinot, O., Boogerd, W., Bravo-Marques, J., Taphoorn, M.J., Kros, J., van de Rijt, C., de Beule, N., and Baron, B. (2003a) EORTC Brain Tumor Study 26972: Second line chemotherapy with Temozolomide in recurrent oligodendroglioma after PCV-chemotherapy: A phase II study. Ann. Oncol. 2003 (in press).

van den Bent, M.J., Looijenga, L.H.J., Langenberg, K., Dinjens, W.N., Graveland, W.J., Uytdewilligen, L., Sillevis Smitt, P.A.E., Jenkins, R.B., and Kros, J.M. (2003b) Chromosomal anomalies in oligodendroglial tumors are correlated to clinical features. Cancer (in press).

Walker, M.D., Alexander, E., Jr., Hunt, W.E., MacCarty, C.S., Mahaley, M.S., Jr., Mealey, J., Jr., Norrell, H.A., Owens, G., Ransohoff, J., Wilson, C.B., Gehan, E.A., and Strike, T.A. (1978) Evaluation of BCNU and/or radiotherapy in the treatment of anaplastic gliomas. A cooperative clinical trial. J. Neurosurg. 49, 333-343.

Walker, M.D., Green, S.B., Byar, D.P., Alexander, E., Jr., Batzdorf, U., Brooks, W.H., Hunt, W.E., MacCarty, C.S., Mahaley, M.S., Jr., Mealey, J., Jr., Owens, G., Ransohoff, J., 2d, Robertson, J.T., Shapiro, W.R., Smith, K.R., Jr., Wilson, C.B., and Strike, T.A. (1980) Randomized comparisons of radiotherapy and nitrosoureas for the treatment of malignant glioma after surgery. N. Engl. J. Med. 303, 1323-1329.

Wallner, K.E., Gonzales, M., and Sheline, G.E. (1988) Treatment of oligodendrogliomas with or without postoperative irradiation. J. Neurosurg. 68, 684-688.

Watanabe, T., Nakamura, M., Kros, J.M., Burkhard, C., Yonekawa, Y., Kleihues, P., and Ohgaki, H. (2002) Phenotype versus genotype correlation in oligodendrogliomas and low-grade diffuse astrocytomas. Acta Neuropathol. (Berl.) 103, 267-275.

Winger, M.J., Macdonald, D.R., and Cairncross, J.G. (1989) Supratentorial anaplastic gliomas in adults. The prognostic importance of extent of resection and prior low-grade glioma. J. Neurosurg. 71, 487-493.

Yung, W.K.A., Prados, M.D., Yaya-Tur, R., Rosenfeld, S.S., Brada, M., Friedman, H.S., Albright, R., Olson, J., Chang, S.M., O'Neill, A.M., Friedman, A.H., Bruner, J., Yue, N., Dugan, M., Zaknoen, S., and Levin, V.A. (1999) Multicenter phase II trial of temozolomide in patients with anaplastic astrocytoma or anaplastic oligoastrocytoma at first relapse. Temodal Brain Tumor Group [erratum in J. Clin. Oncol. 17, 3693, 1999]. J. Clin. Oncol. 17, 2762-2771.

Yung, W.K.A., Albright, R.E., Olson, J., Fredericks, R., Fink, K., Prados, M.D., Brada, M., Spence, A., Hohl, R.J., Shapiro, W., Glantz, M., Greenberg, H., Selker, R.G., Vick, N.A., Rampling, R., Friedman, H., Phillips, P., Bruner, J., Yue, N., Osoba, D., Zaknoen, S., and Levin, V.A. (2000) A phase II study of temozolomide vs. procarbazine in patients with glioblastoma multiforme at first relapse. Br. J. Cancer 83, 588-593.

Zlatescu, M.C., TehraniYazdi, A., Sasaki, H., Megyesi, J.F., Betensky, R.A., Louis, D.N., and Cairncross, J.G. (2001) Tumor location and growth pattern correlate with genetic signature in oligodendroglial neoplasms. Cancer Res. 61, 6713-6715. 